Review article

\title{
Image-guided left ventricular lead placement in cardiac resynchronization therapy: focused on image fusion methods
}

\author{
Premysl Hajek ${ }^{1 *}$, Iva Safarikova ${ }^{1,2}$, Jan Baxa ${ }^{3}$ \\ ${ }^{1}$ Ceske Budejovice Hospital, Department of Cardiology, Ceske Budejovice, Czech Republic \\ ${ }^{2}$ University of South Bohemia in Ceske Budejovice, Faculty of Health and Social Sciences, Budejovice, Czech Republic \\ ${ }^{3}$ Charles University in Prague, University Hospital and Faculty of Medicine in Pilsen, Department of Imaging Methods, Pilsen, Czech Republic
}

\begin{abstract}
Cardiac resynchronization therapy is an effective and widely accessible treatment for patients with advanced, drug-refractory heart failure. It has been shown to reverse maladaptive ventricular remodeling, increase exercise capacity, and lower hospitalization and mortality rates. However, there still exists a considerable proportion of patients who do not respond favorably to the therapy. Tailored left ventricular (LV) lead positioning instead of empiric implantation is thought to have the greatest potential to increase response rates. In our paper, we focus on the rationale for guided LV lead implantation and provide a review of the non-invasive imaging modalities applicable for navigation during LV lead implantation, with special attention to the latest achievements in the field of multimodality imaging and image fusion techniques. Current limitations and future perspectives of the concept are discussed as well.
\end{abstract}

Keywords: Cardiac resynchronization; Coronary sinus; Guided implantation; Image fusion; Mechanical activation; Multimodality imaging; Myocardial scar; Non-responder

\section{Highlights:}

- Despite its general efficacy, cardiac resynchronization therapy fails in 30-50\% of patients.

- Tailored left ventricular lead implantation is considered the most promising measure to take full advantage of the therapy.

- Conventional imaging provides limited information; only multimodality imaging and image-fusion provide effective guidance.

- With alternative approaches to resynchronization, there is an urgent need for comprehensive imaging in clinical practice.

\begin{abstract}
Abbreviations:
2D, two-dimensional; 2Y, 2-year follow-up; 3D, three-dimensional; 3D-RTE, three-dimensional real time echocardiography; 6M, 6-month follow-up; AHR, acute hemodynamic response; AV, anterior veins of the left ventricle; CMR, cardiac magnetic resonance; CRT, cardiac resynchronization therapy; CS, coronary sinus; CT, computed tomography; CX, circumflex artery; EDV, end-diastolic volume; EF, ejection fraction; ESV, end-systolic volume; HF, heart failure; ICM, ischemic cardiomyopathy; LA, left atrium; LAD, left anterior descending artery; LAO, left anterior oblique; LBBB, left bundle branch block; LEAA, latest electrically activated area(s); LGE, late gadolinium enhancement; LMAS, latest mechanically activated segment(s); LV, left ventricle/ventricular; LVESV, left ventricular end-systolic volume; MDCT, multi-detector computed tomography; MPI, myocardial perfusion imaging; MRI, magnetic resonance imaging; PET, positron emission tomography; RA, right atrium; RAO, right anterior oblique; RCT, randomized, controlled trial; RV, right ventricle/ventricular; SDI, systolic dyssynchrony using endocardial tracking of CMR cine images in short and long axis; SPECT, single photon emission computed tomography; SQUEEZ, Stretch Quantifier of Endocardial Engraved Zones; SSFP, steady state free precision; STE, speckle tracking echocardiography; TEE, transesophageal echocardiography
\end{abstract}

\section{Introduction}

Since the first resynchronization device became commercially available in the United States in 2001, cardiac resynchronization therapy (CRT) has evolved into an effective and widely accessible treatment option for patients with drug-refractory heart failure (HF) (Abraham et al., 2002). Despite advances in technology and implantation techniques, there still exists a substantial proportion of patients who do not respond favorably to the therapy in terms of functional improvement or reversal of maladaptive remodeling, with the latter being emphasized as the only reliable surrogate metric for assessing clinical outcomes (Groenning et al., 2000). The reported rates of these non-responders vary depending on the criteria used but are estimated to be $30-50 \%$ (Auricchio and Prinzen,

\footnotetext{
* Author for correspondence: Premysl Hajek, Ceske Budejovice Hospital, Department of Cardiology, B. Nemcove 585/54, 37001 Ceske Budejovice, Czech Republic; e-mail: premek.hajek@email.cz http://doi.org/10.32725/jab.2019.019

Submitted: 2019-08-18 • Accepted: 2019-10-30 • Prepublished online: 2019-11-13

J Appl Biomed 17/4: 199-208 • EISSN 1214-0287 • ISSN 1214-021X

(c) 2019 The Authors. Published by University of South Bohemia in České Budějovice, Faculty of Health and Social Sciences.

This is an open access article under the CC BY-NC-ND license.
} 
2011). Strategies for achieving higher response rates focus on several issues: candidate selection, optimal device programming, and appropriate left ventricular (LV) lead positioning. Among these, improving the latter is perceived as having the greatest potential benefits (Daubert et al., 2017).
In this paper, we provide a review of multimodality imaging and image fusion techniques applicable for implantation navigation (Fig. 1).

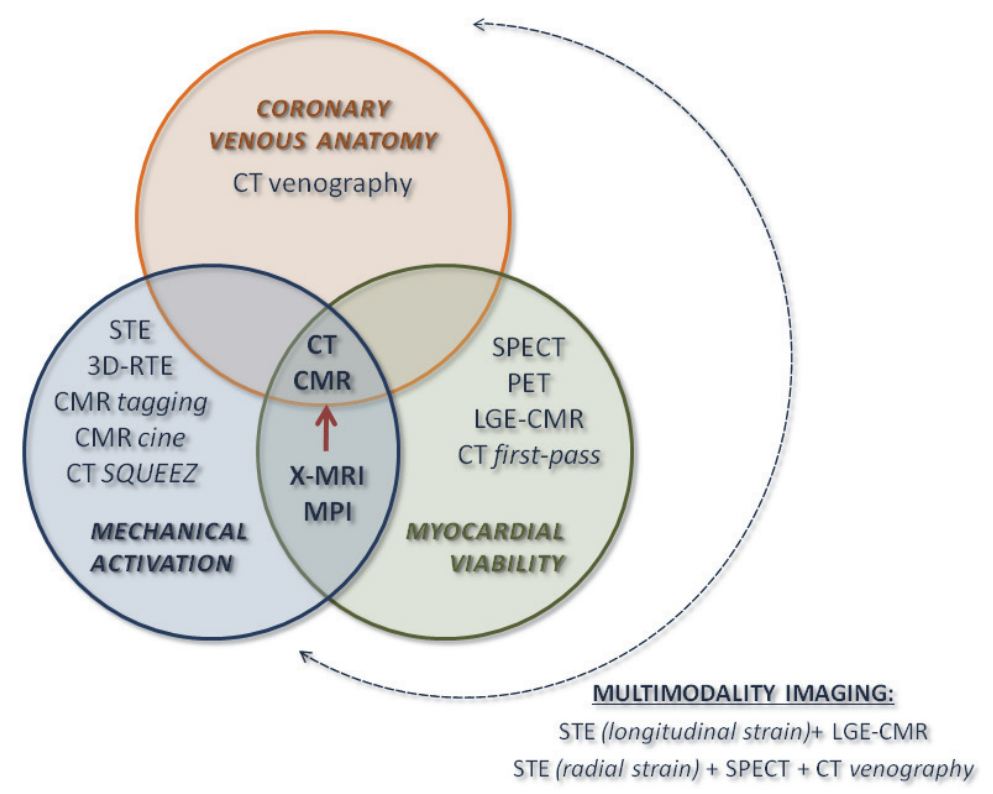

Fig. 1. Comprehensive imaging in CRT implantation. Each circle represents an individual task for the preoperative imaging, with applicable modalities listed. In the centre, there are methods that provide multiple pieces of information from a single study: CT and CMR, albeit the latter provides only low quality delineation of the CS tributaries. X-MRI is an example of technology that enables fusion of a CMR-derived 3D model with fluoroscopy venograms. The fusion of venograms with MPI-derived models is also clinically applicable. Distinctive combinations of modalities evaluated in clinical trials are as well mentioned (multimodality imaging).

\section{Current approach to CRT-delivery and rationale for guided implantation}

Limitations to empiric left ventricular lead implantation The prerequisite for the restoration of energetically efficient contraction is compensation for altered electrical wave propagation through the ventricles. This can be effectuated by simultaneous stimulation of the right ventricle (RV) and the latest electrically activated area (LEAA) of the LV (Abraham et al., 2002). Findings from early hemodynamic studies as well as randomized, controlled trials suggest that the lateral and posterolateral aspects of the mid-ventricular or basal portions of the LV, supposedly harboring the LEAAs, are optimal for LV pacing (Brignole et al., 2013; Butter et al., 2001; Dekker et al., 2004; Kutyifa et al., 2013; Singh et al., 2011). With more data available, however, it has become obvious that this guidelines-endorsed approach does not work for all CRT recipients (Dekker et al., 2004).

\section{Major determinants for the effective resynchronization}

The overall myocardial scar burden as well as high scar density correlates inversely with LV functional improvement and are predictive of worse outcomes (Adelstein and Saba, 2007; Adelstein et al., 2011; Ypenburg et al., 2007a). This may result from the paucity of contraction-capable tissue but is mainly caused by impaired electrical wave front propagation (Lambiase et al., 2004). Scar distribution with respect to LV lead position is also of considerable importance (Chalil et al., 2007). By non-contact endocardial mapping, areas with low amplitude signals and low conduction velocities were identified (Ypenburg et al., 2007b). Pacing at these sites that corresponded to scarred myocardium and actually represented LEAAs, was associated with QRS prolongation and it failed to restore synchronicity unless the LV stimulus preceded the RV stimulation by 3040 ms (Bleeker et al., 2006; Ypenburg et al., 2007b).

In a study of 559 patients, Leyva et al. (2011) used late gadolinium enhancement cardiac magnetic resonance (LGECMR) in 209 to guide LV lead placement away from fibrotic tissue. Compared with patients paced at viable myocardium, patients in whom the LV lead was placed over scarred tissue had the highest risk of cardiovascular death $(\mathrm{HR}=6.34)$, as well as the composite endpoint of all-cause death or hospitalization for major cardiac events $(\mathrm{HR}=4.74)$. In the non-scar paced group, a scar tissue burden of less than the cut off value of $10 \%$ was predictive of better clinical outcomes. In addition, fibrosis of the segments adjacent to the target segment may also interfere with a favorable response (Adelstein et al., 2011; Becker et al., 2011).

Since it is not feasible, in routine practice, to determine electrical wavefront propagation and LEAAs non-invasively, the mechanical activation sequence, traced by echocardiography-based methods, CMR, or myocardial perfusion imaging (MPI), serves as a surrogate for the electrical potential spread. Available evidence is derived mainly from two randomized, controlled trials - TARGET and STARTER (Khan et al., 2012; Saba et al., 2013) - that used 2-dimensional (2D) speckle tracking echocardiography (STE) radial strain analysis to guide LV lead implantation towards the late mechanically activated segment (LMAS). In the TARGET trial, the guided implantation was associated with a reduction of LV end-systolic volume (LVESV) by $>15 \%$ in $70 \%$ of patients, whereas in the standard-implantation group, it was seen in only $55 \%$ of 
cases. This implies that about 7 patients have to undergo guided implantation to gain one additional responder (Khan et al., 2012). In the STARTER trial (Saba et al., 2013), patients assigned to guided implantation experienced less clinical events (combined endpoint of all-cause death and HF-related hospitalization) by $26 \%$ at the 2 -year follow-up. This was driven mainly by a reduction in the hospitalization rate $(\mathrm{HR}=0.48$, $p=0.049$ ). None of the trials showed a benefit for guided LV lead implantation relative to mortality.

MPI by single-photon emission computed tomography (SPECT) or positron emission tomography (PET) represents an accessible alternative to echocardiography-based approaches. The tracer (thallium-201 or technetium-99m in SPECT, 18-fluorodeoxyglucose in PET) count-variation temporal analysis produces time-activity curves for each sample volume, permitting a phase-polar map of mechanical activation to be constructed (Phase Analysis Technique) (Chen et al., 2008).

Besides the myocardial viability and activation pattern, suitable coronary venous anatomy is an essential precondition for successful guided (as well as non-guided) LV lead delivery.
The number and trajectories of coronary veins are acknowledged to be highly variable, with an average of $2-5$ potentially suitable coronary sinus (CS) first-order tributaries, i.e., those of acceptable diameter, subtending the posterolateral aspects of LV, most often recognized by the authors - Fig. 2 (Christiaens et al., 2008; Jongbloed et al., 2005; Spencer et al., 2014; Van de Veire et al., 2006). There are also studies demonstrating that more than one suitable vein is present in less than half of the patients undergoing CRT implantation (Khan et al., 2009), and in almost one-third, no suitable vein is found that subtends the empirically optimal regions (Spencer et al., 2014). Without preoperative imaging, the actual number and course of the vessels remain unknown to the implanter until the CS is cannulated and a contrast venogram is obtained, which precludes an optimal strategy being determined prior to the procedure. A comprehensive evaluation of the coronary venous system with computed tomography (CT) would help to assess the patient's suitability for transvenous implantation and predict technical difficulties that might arise from unfavorable anatomy (Fig. 2).

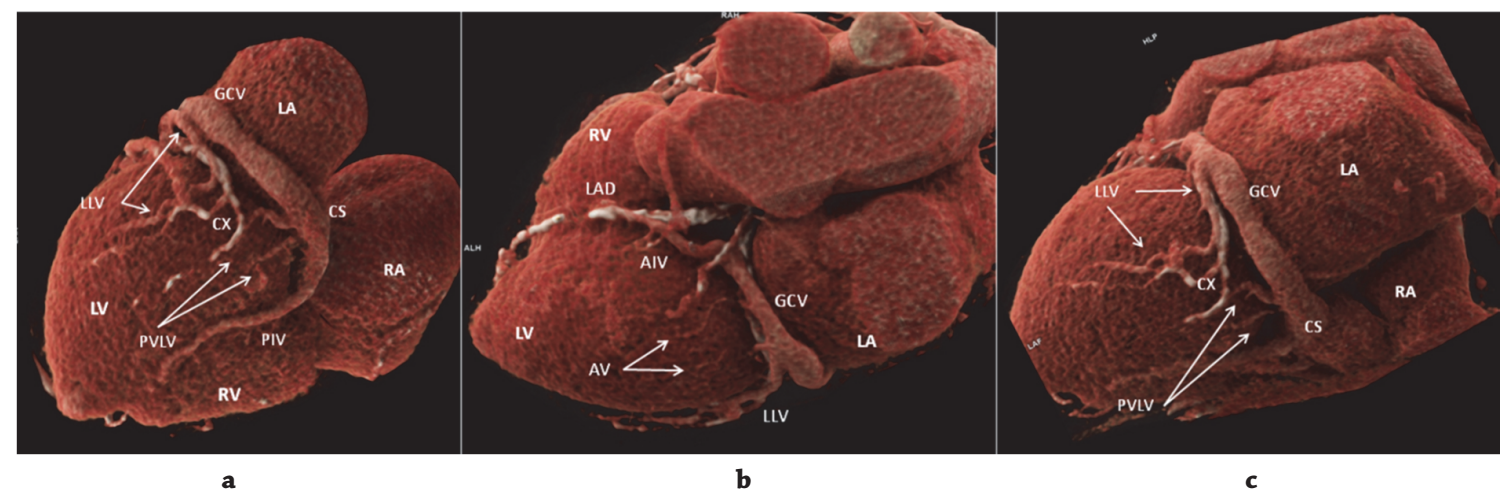

Fig. 2. 3D reconstruction of the coronary venous system. Posterolateral (a) and superior (anterolateral aspect of the left ventricle) (b) views. The first tributary of the coronary sinus (CS) is the posterior interventricular vein (PIV, or middle cardiac vein), running in the posterior interventricular groove. In this example, the posterior veins of the left ventricle (PVLV) are represented by a couple of subtle tributaries, not suitable for lead deployment. Note the acute angulation (on the lateral view (c) which may preclude successful deployment of the lead into the left lateral vein (LLV) that subtends the empirically optimal posterolateral aspect of the LV. The great cardiac vein (GCV) continues as the anterior interventricular vein (AIV) in the anterior interventricular groove.

\section{Comprehensive imaging to facilitate optimal site selection during CRT}

It is obvious that a combination of imaging modalities is needed for effective and unrestricted guidance. Generally, three distinct concepts of comprehensive imaging have been proposed and evaluated: multimodality imaging, methods deriving all the relevant information from single imaging study (one-stop shops), and real-time image fusion (Fig. 1).

\section{Multimodality imaging}

Multimodality imaging refers to the complementary use of mostly echocardiography-based methods to define the LMAS, plus one or more other imaging modalities to provide information on tissue viability and/or coronary venous anatomy. The preoperatively obtained data can be put together to determine the target position, which is usually displayed on a polar plot map. The implantation itself is, however, performed using a standard, plain fluoroscopic control, with the polar plot map serving as only indirect navigation tool.

In an observational study of 100 consecutive patients (Bertini et al., 2016), STE longitudinal strain was used to define the most delayed segment and LGE-CMR to avoid fibrosis. At 6 months, $78 \%$ of patients from the guided group reverse-re- modeled, compared to $56 \%$ in the standard-implantation group and $54 \%$ vs. $32 \%$ of the patients were classified as super-responders (LVESV reduction $\geq 30 \%$ ). Of note, quadripolar leads were used more often in the guided group (64\% vs. $10 \%)$.

In a randomized, controlled trial, Sommer et al. (2016) evaluated the superiority of guidance towards the LMAS separate from scar, as defined by STE radial strain and SPECT, respectively. This was supplemented by a $3 \mathrm{D}$ CT reconstruction of the coronary venous system $(n=89)$. Notably, in the control group $(n=93)$, the "routine" CRT implantation was guided by left ventricular electrical delay (QLV). As a result, the distribution of concordant and adjacent LV leads was comparable between groups (99\% vs. 98\%) as well as QLV values. The rate of non-response, mainly in terms of failure to improve NYHA class or to increase the six-minute walk distance, was $26 \%$ in the guided group compared to $42 \%$ in the control group (OR for non-response $=2.29 ; p=0.02$ ). No difference was observed regarding the combined end-point of all-cause death and HF-hospitalization or reverse remodeling.

One-stop imaging shops for CRT-guidance

The most promising alternative to multimodality imaging is cardiac CT. Nuclear imaging and CMR are also capable of pro- 
viding multiple pieces of information from a single imaging study, albeit the visualization of CS branches is of lower quality (Ma et al., 2012; Zhou et al., 2014).

Conventional viability assessment using cardiac CT relies on a similar premise as CMR, i.e., the altered distribution of primarily extracellular, interstitial contrast agent with comparable kinetics (iodine as well as gadolinium). However, apart from being highly susceptible to inadequate contrast agent dosing, late-enhancement imaging fails to reliably delineate chronic scarring. In this context, evaluation of relative hypoperfusion on the first-pass contrast scan and local wall thickness <6 mm has been shown (Behar et al., 2017a; Mendoza et al., 2010) to be more sensitive. Current CT scans enable the analysis of regional wall deformation by tracing endocardial-surface features (trabeculae), depicted inversely as a surface of contrast-enhanced 3D blood cast. Following image acquisition, an endocardial-surface mesh consisting of multiple triangular patches is generated. Displacement of the vertices in each of the patches is tracked over the cardiac cycle, which allows changes in the surface area to be calculated and expressed using a metric called SQUEEZ (Stretch Quantifier of Endocardial Engraved Zones) (Pourmorteza et al., 2012). Regional time delay to peak SQUEEZE-derived strain for each segment is measured to define the LMAS with a temporal resolution of 70-100 ms. (For comparison, echocardiography and CMR provide resolution $20 \mathrm{~ms}$ and 35-50 ms, respectively.) The tissue is considered viable if the SQUEEZ amplitude $>10 \%$. Deploying the LV lead into the vein subtending the CT-derived target in 18 patients who were subjects to a CRT upgrade with an existing pacing system resulted in an AHR rate of $92 \%$ (a $10 \%$ increase in contractility $\left(\mathrm{LVdP} / \mathrm{dt}_{\max }\right)$ ). In $70 \%$ of cases, the CT-SQUEEZ target corresponded with the site of the best achievable AHR of all eligible venous targets. At 6 months, clinical improvement was seen more often (90\% vs.
$60 \%$ ) in patients paced at CT-SQUEEZ targets (Pourmorteza et al., 2012).

\section{Image fusion techniques}

Simple visual correspondence was used in the landmark TARGET and STARTER trials (and the majority of others, including the discussed multimodality imaging studies) to match the bi-plane fluoroscopic view with the respective echocardiographic projection. This approach inevitably leads to inaccurate alignment of the coronary veins with the myocardial surface (Fig. 3). As demonstrated in a study with CT, the overall agreement with the lead position classification, on the basis of fluoroscopy, was only $35 \%$ for LV leads and only $22 \%$ for RV leads (Sommer et al., 2014). Interobserver agreement on LV and RV lead positions was poor, and the situation may be even worse in patients with extensively remodeled and rotated hearts (Singh et al., 2005). Partly due to these constraints, target-lead concordance was achieved in only a limited number of patients in the principal trials (64\% and $30 \%$ for TARGET and STARTER, respectively, as an example) (Khan et al., 2012; Saba et al., 2013). On the contrary, co-registration of the 3D echocardiography-derived endocardial shell, containing information on wall motion, with the $3 \mathrm{D}$ reconstruction of the coronary venogram, obtained through high-speed rotation angiography in 37 subjects to CRT implant, the concordance rate of $97 \%$ was achieved (Döring et al., 2013; Tournoux et al., 2007).

A precursor of real-time image integration is represented by a simultaneous projection of a LGE-CMR derived interactive 3D, color-coded, surface-rendered navigational model and the matched fluoroscopic view (Laksman et al., 2014). Analyzing the data according to a pre-specified algorithm, the software provided information about the suitability of individual segments for both RV and LV lead deployment: the RV lead was placed toward the apex (or away from scar), with the tar-

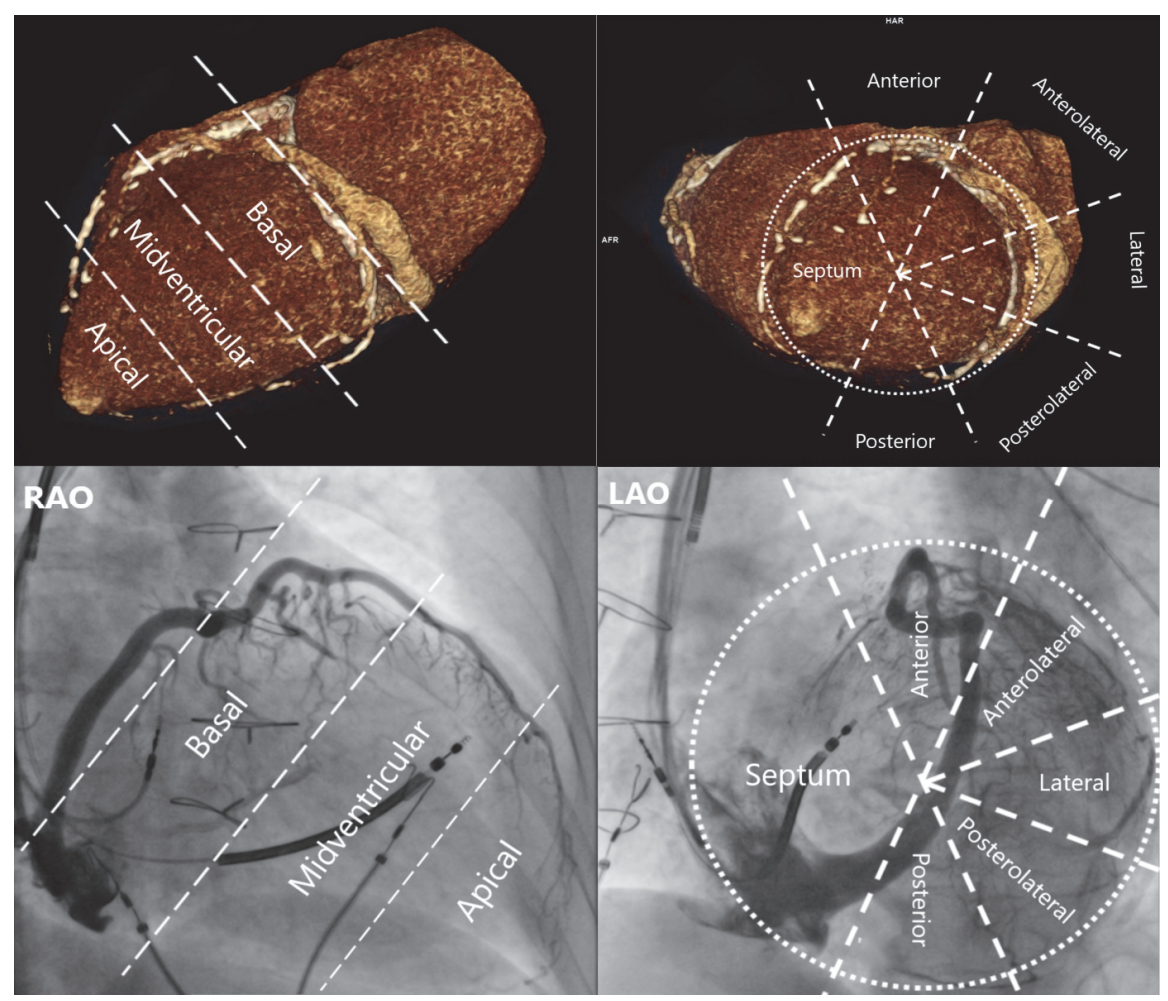

Fig. 3. An example illustrating the imprecision of $2 \mathrm{D}$ visualization of $3 \mathrm{D}$ structures. Coronary venous reconstruction with common left ventricular segment classification and corresponding 2D fluoroscopic views. 
get for the LV lead being determined by assessing (1) the lowest local scar burden, (2) the greatest mechanical delay, and (3) the distance from the RV lead. With this approach, the LV lead was successfully delivered to the prescribed or immediately adjacent segment in $97 \%$ of cases (30 of 31 patients). Reverse remodeling criteria (LVESV reduction $215 \%$ ) were met in $74 \%$ of patients, while $58 \%$ met the super-response criteria (LVESV reduction $>30 \%$ ) (Laksman et al., 2014).

The feasibility of fusing a 2D fluoroscopic view with 3D CMR-derived data regarding cardiac anatomy (i.e., myocardial surface segmentation, scar distribution, and transmurality), and coronary venous anatomy along with information on the LMAS was tested in a cohort of 21 subjects (Ma et al., 2012). Whereas a model of all four chambers was created automatically, reconstruction of the CS required manual segmentation. The cine steady-state free precision (SSFP) sequences were performed for motion analysis on the basis of regional volume changes, and delayed-enhancement imaging for automated scar delineation. The $3 \mathrm{D}$ roadmap was manually registered to live X-ray data using multiple views of a catheter looped in the right atrium and a lead in the RV as registration features, ensuring correct spatial orientation. The alignment of the overlay was maintained automatically throughout the procedure. Nevertheless, guidance based on the $3 \mathrm{D}$ image projected as a $2 \mathrm{D}$ mono-plain X-ray view does not allow the actual position of the LV lead to be easily appreciated. To overcome the constraints inherent to plain 2D visualization of a 3D structure, the software transforms the $3 \mathrm{D}$ roadmap into a classic 2D bull's eye plot, depicting all the information critical for comprehensive navigation, including the principal tributaries of the CS and the actual lead position. However, the CMR-derived visualization of the distal CS branches was of lower quality, particularly in the presence of atrial fibrillation or ectopic beats. Provided the image quality was satisfactory; a contrast venogram was not required for implantation (Ma et al., 2012).

The effectiveness of the method on invasively measured AHR and LV reverse remodeling was assessed by Shetty et al. (2013). Leads were implanted in target segments in 15 of 20 patients. Compared to an acute response rate of $50 \%$ with empiric navigation, CMR-based guidance was not associated with a significant increase in the acute response rate (60\%). However, in only 2 of the 8 patients who failed to acutely re- spond despite being paced at the CMR-target, any position related to better hemodynamic response existed. Of the patients who showed $>15 \%$ LVESV reduction at the 6 -month follow-up (60\%), 92\% were paced in the target segment, while this was the case in only $50 \%$ of non-responders. Conversely, $50 \%$ of the non-responders did not experience reverse remodeling despite being paced in the preferred segment. Interestingly, AHR did not necessarily predict reverse-remodeling at 6 months, since it was achieved in only $71 \%$ of acute responders.

The need for further work-flow optimization resulted in the development of the X-MRI facility that incorporates MRI scanner and a biplane angiography suite, connected to a dedicated workstation (Behar et al., 2017b). The key feature represents an integrated software platform designed for rapid processing of comprehensive CMR data that is displayed as a 3D model on a biplane fluoroscopy screen. The whole process of analyzing the data and creating the 3D model is fully automated, with an opportunity for the clinician to adjust the results after each step (anatomical segmentation, scar distribution, transmurality and burden, mechanical activation, and LMAS identification; CS anatomy is not part of the reconstruction), and, more importantly, the process is completed while the patient is being transferred from the MRI to the electrophysiology laboratory and is being prepared for implantation (Fig. 4). The 3D model is unfolded into a color-coded 16-segment model bull's-eye plot and the target segment selected by the physician (Fig. 5). Once the CS is cannulated, the epicardial and endocardial shells are fused with the occlusive coronary venogram (Fig. 6).

Whereas in the preceding settings the CMR needs to be performed days or even weeks before the implantation owing to lengthy computational processing, the capacity of the current system permits the implantation to immediately follow image acquisition. Nevertheless, even with this cutting-edge technology, only $71 \%$ of the leads were deployed over the target segments. The rest of them were positioned at or adjacent to scarred tissue due to a lack of viable CS tributaries. It is noteworthy in this context that the detailed scar visualization allowed for the poles of multipolar leads to be directed away from fibrosis. The result was a mean decrease in QRS length of $25 \mathrm{~ms}$ (significant), but, being a feasibility study, it lacks any outcome evaluation (Behar et al., 2017b).
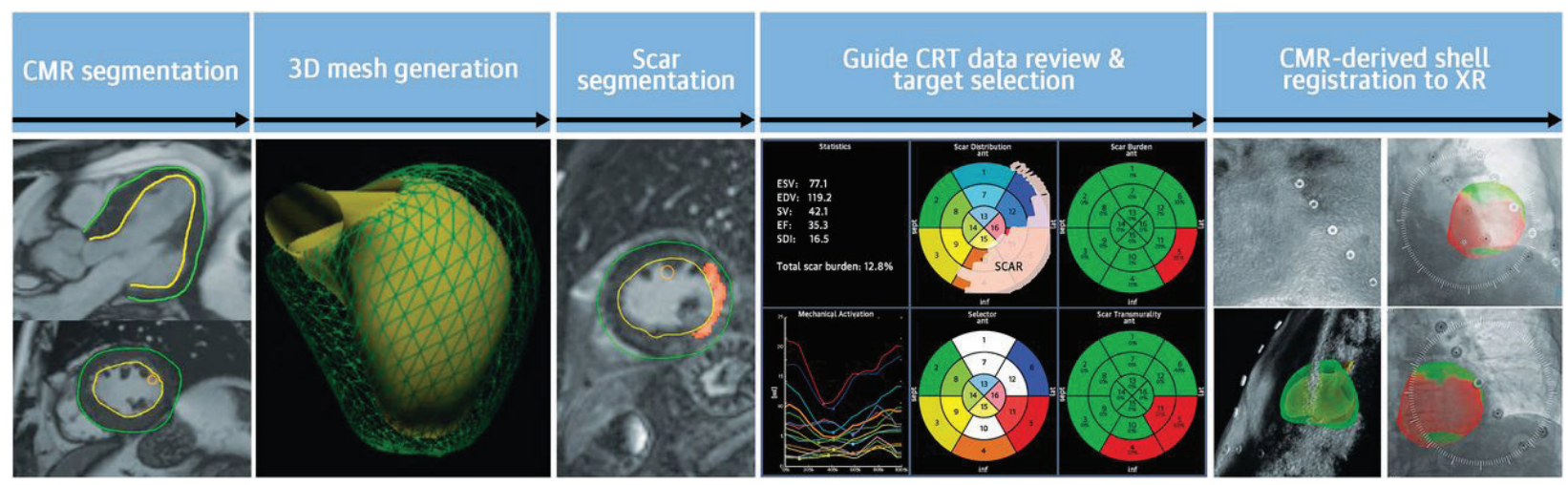

Fig. 4. X-MRI imaging workflow. The first step involves the automated segmentation and manual adjustment of the epicardial and endocardial slices to generate a 3-dimensional (3D) mesh. Endocardial wall motion is tracked over the cardiac cycle to generate volume vs. time curves for the 16 segments. Following registration of the cine to late gadolinium enhancement sequences, areas of myocardial fibrosis are identified, and both scar and dyssynchrony data are reviewed and targets selected. The $3 \mathrm{D}$ shell is then registered to the $\mathrm{x}$-ray (XR) once the patient is on the catheter laboratory table using fiducial markers, which show up white (from the CMR sequences) and gray with a central dark dot (lead ball-bearing) on the x-ray. Vertical and horizontal translation using biplane fluoroscopy is used, in addition to rotation about the $\mathrm{x}, \mathrm{y}$, and $\mathrm{z}$ axes to line up the markers as demonstrated on the right hand panel. Following this process, the CMR-derived 3D model is registered to the XR system and every fluoroscopic cine demonstrates the epicardial and endocardial shell overlaid in the correct orientation.

Reproduced with permission from Elsevier due to the Creative Common License (Behar et al., 2017b). 


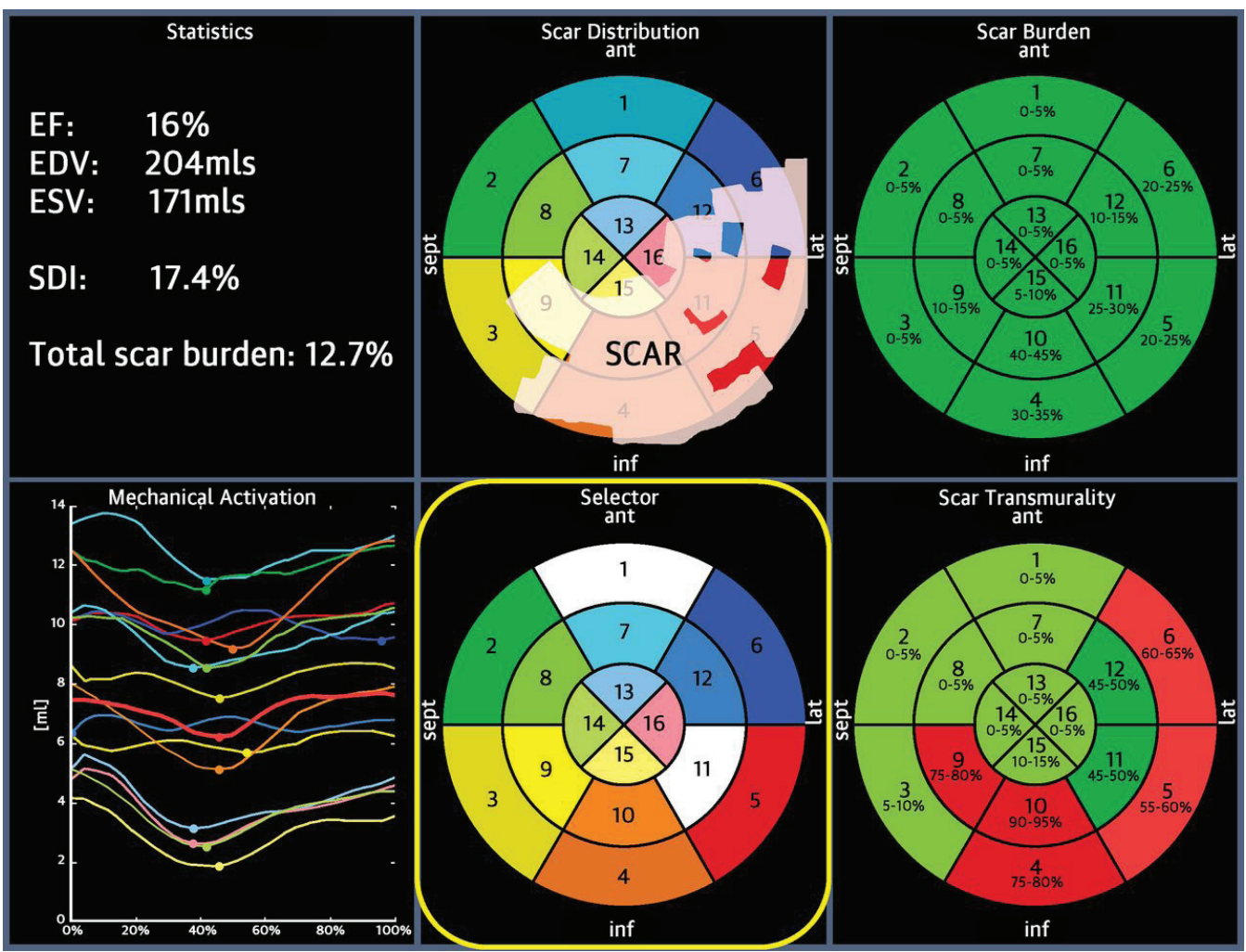

Fig. 5. Processing of the MRI dataset. This display screen is seen following the processing of the CMR dataset and is mimicked on the large screen in the catheter laboratory. Total scar burden calculated as a mean of all myocardial segments. (Top middle) Scar distribution denoted in gray upon an American Heart Association 16-segment model. (Top right) Scar burden (\% scar per myocardial segment volume), displayed in 5\% ranges.

(Bottom right) Scar transmurality demonstrating the mean transmurality from endocardium to epicardium. Those segments $>50 \%$ transmural myocardial fibrosis are also denoted in red. (Bottom left) Mechanical activation curves for the 16 segments, corresponding to the colors shown in the middle panels. Endocardial tracking of the left ventricle provides absolute changes in the volume per segment (ml, y axis) over the cardiac cycle. Because these are absolute volume changes, the apical segments are always at the bottom because they have a smaller start and end volume. When the user hovers over a segment in the top middle panel, the associated volume time curve appears in bold; in this case, the target posterolateral segment is shown. (Bottom middle) Target selection panel. Upon reviewing the scar location, burden, transmurality, and mechanical activation curves, target segments are chosen (seen here in white; basal anterior, mid-posterolateral).

Reproduced with permission from Elsevier due to the Creative Common License (Behar et al., 2017b).
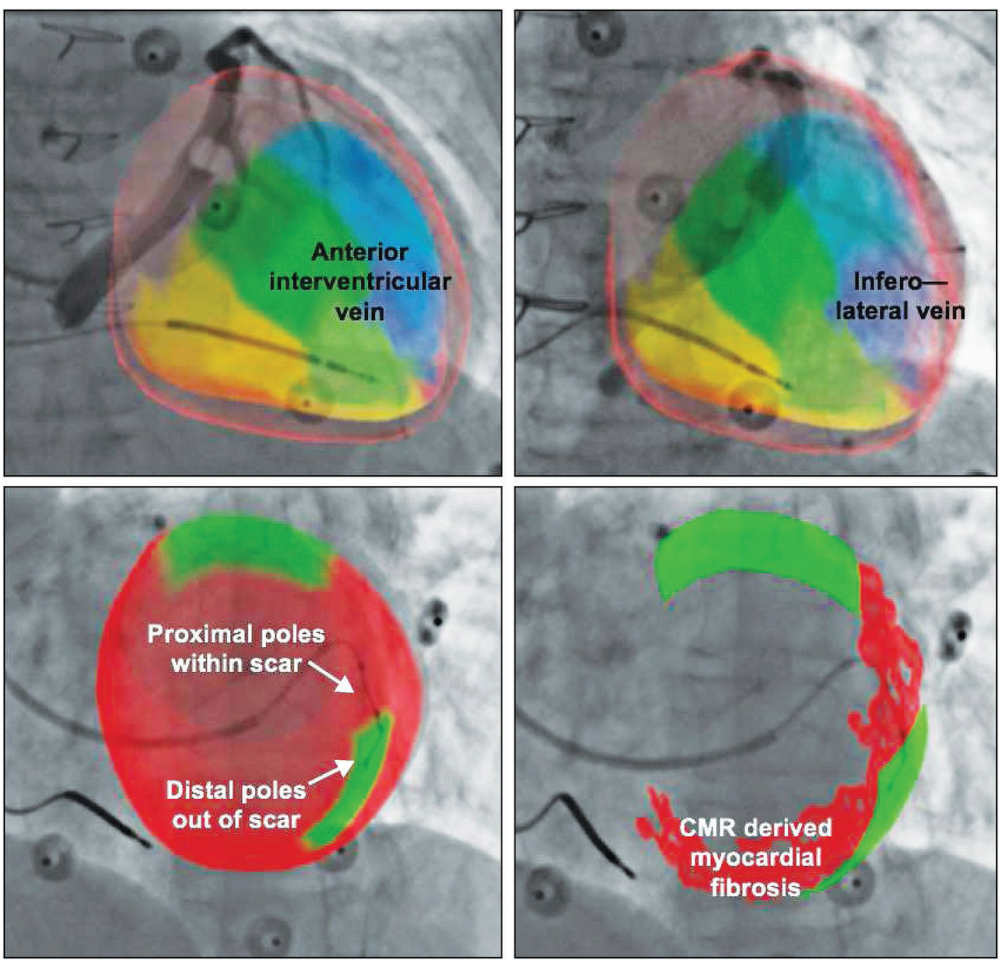

Fig. 6. CMR-Derived Image Overlay With Target Segment Selection. (Top left) Anteroposterior venogram with overlay of CMR-derived epicardial/endocardial shell with 16-segment American Heart Association model showing an anterior interventricular vein. The 3D CMR-derived shell has the same colors as displayed in the guidance platform as shown in Supplemental Figures 1 a 2. Inferoseptal, anteroseptal, and anterior segments are colored in yellow, green, and blue, respectively. (Top right) left anterior oblique (LAO) 20 venogram with automated rotation and alignment of the 16-segment model with the $\mathrm{x}$-ray. Inferolateral veins are demonstrated. (Bottom left) LAO 40 projection. Positioning of a quadripolar left ventricular lead into a preselected target segment (green). (Bottom right) LAO 40 projection, alternate view with CMR-derived scar distribution (red). Attempted positioning and pacing using left ventricular poles out of regions of scar.

Reproduced with permission from Elsevier due to the Creative Common License (Behar et al., 2017b). 
The considerable overlay accuracy of a 3D epicardial surface on a SPECT MPI with 3D venous anatomy, reconstructed from dual-view fluoroscopy venograms, was demonstrated by Zhou et al. (2014). Following manual identification of the major coronary veins on dual-view fluoroscopy venograms, vessel bifurcations served as fiducial markers for a 3D recon- struction of the venous tree and interventricular grooves on a 3D SPECT image as landmarks for proper surface-vein alignment (Fig. 7). The overall distance-based mismatch error between the fluoroscopic and contrast-enhanced CT venograms registered on SPECT images was $4.6 \pm 3.6 \mathrm{~mm}$ (range 0 to $16.9 \mathrm{~mm}$ ).
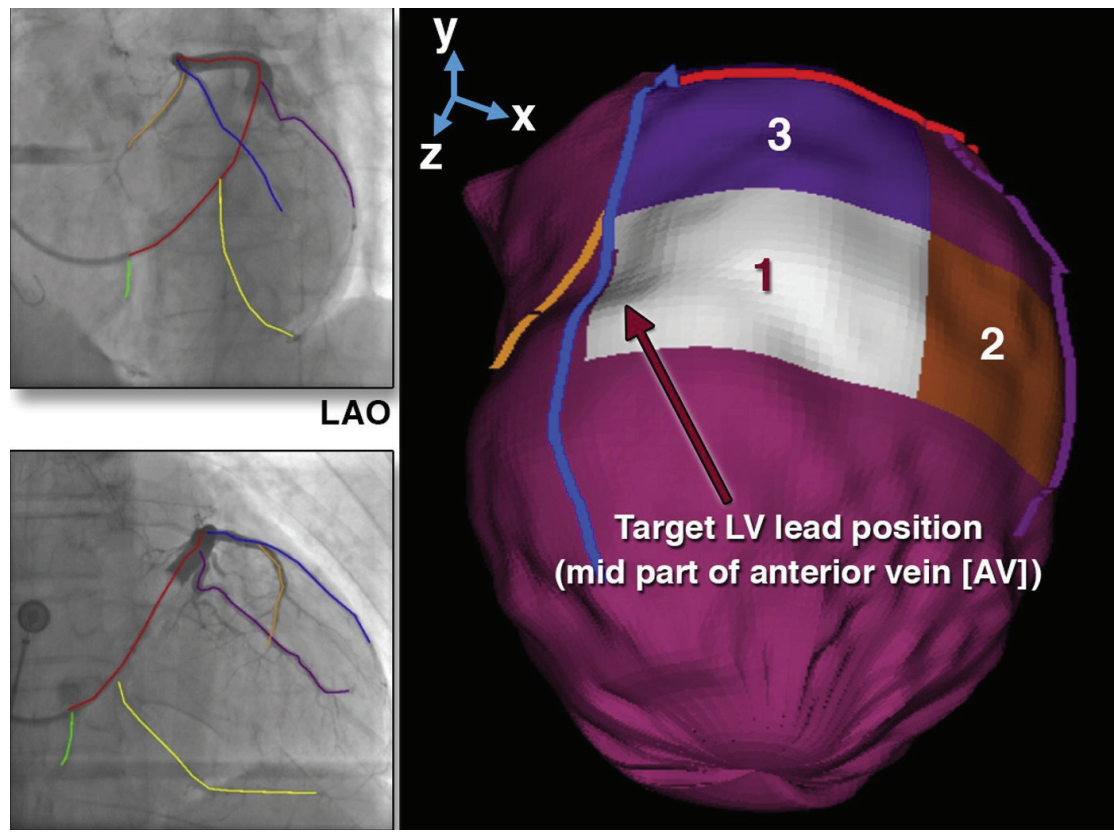

Fig. 7. SPECT-Vein Navigation Tool Kit. Major left ventricular (LV) veins were drawn on fluoroscopic venograms, reconstructed to a 3D structure, and fused with a SPECT LV epicardial surface. The mid part of the anterior vein (blue line) was aligned with the optimal segment (white).

Reproduced and adapted with permission from Elsevier (Zhou et al., 2014).

Ludwig et al. (2015) recently presented technique that merges SPECT-derived data regarding viability and activation timing with $\mathrm{CT}$ images to create a comprehensive 3D surface-rendered model. Anatomically discrete regions of the coronary venous system were used as a fiducial system: by matching the MDCT venogram with a virtual venogram obtained through continuous recording of the mapping catheter's spatial location, an accurate registration of the SPECT-MDCT fusion image is ensured. MDCT also reliably depicts the course of left phrenic nerve over the epicardial surface. The method was found to be feasible, with acceptable procedural times.

\section{Applicability of advanced imaging techniques in a clinical practice}

The concept of guided CRT implantation sounds appealing particularly in specific subgroups of patients with ICM and/ or a borderline surface ECG (QRS 130-150 ms, a non-specific ventricular conduction disturbance). Studies so far have consistently demonstrated the beneficial effect on functional, hemodynamic, and echocardiographic parameters when individual tasks, i.e., pacing an LMAS that is free from fibrosis, are accomplished. Few randomized trials have provided evidence on favorable clinical outcomes. Table 1 summarizes the results of principal trials in terms of navigation success rates and clinical outcomes in comparison with control groups. Nonetheless, when the analyses are performed according to the intention-to-treat principle, the results suddenly lose their once impressive shine. Reasons for this may be as follows: (1) 10 to $15 \%$ of patients considered for CRT are not eligible for the imaging study due to contrast agent allergy, renal disease, claus- trophobia, etc., (2) in another $10 \%$ of patients, unsatisfactory image quality prevents any advanced analysis, and, above all, (3) the preferred or neighboring segments are reached in only about two-thirds of patients, mainly due to lack of suitable venous tributary or because of technical constraints (i.e., lead instability, inadequate pacing threshold, phrenic nerve stimulation, etc.).

Apart from higher costs, other issues also come together to keep the concept from being adopted into clinical practice:

- The vast majority of the advanced modalities utilize purpose-built facilities and software platforms installed on workstations specially developed to investigational purposes. As such, they are currently not commercially available.

- Data post-processing and evaluation require specially trained clinicians, equipped with skills and expertise greatly exceeding routine practice standards.

- Reproducibility of findings derived from these imaging studies is limited by a substantial inter-individual variability unless the data are analyzed in a fully automated process; notably, these are the most accessible modalities (e.g., echocardiography-based) that are particularly prone to misinterpretation.

- Data acquisition, synthesis, and interpretation are hugely time-consuming processes. Therefore, for the majority of centers outside of research institutions, it would not be feasible to perform the pre-implantation studies on a routine. Broadening the spectrum of cardiac conditions submitted to imaging may, however, offer a possible solution: CMR is currently a cornerstone in the evaluation algorithm 


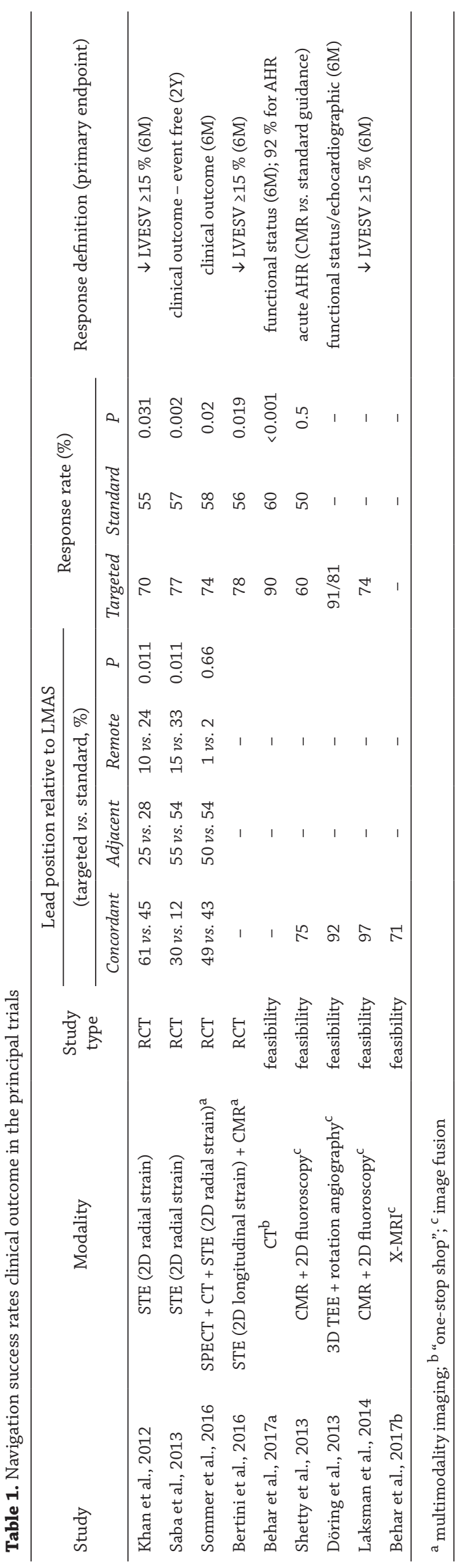

for severe myocardial dysfunction, so are cardiac $\mathrm{CT}$ and MPI for the non-invasive diagnosis of coronary artery disease in selected patients. Adjustments of current protocols can yield the desired information. Remarkably, a coronary venogram can be easily obtained at the venous phase of coronary angiography without the need of additional contrast medium, and with only a slight increase in radiation exposure!

- Even when successfully adopted into practice, image-guided CRT implantation would inevitably give rise to many important questions, e.g.: What should be the strategy once no suitable coronary vein is detected? What should be the acceptable distance from the target segment and when should the patient be assigned to epicardial surgery, which permits site-selective LV pacing? What scar burden threshold (and other fibrosis characteristics) should be therapy exclusion criteria for patients otherwise eligible according to the current clinical practice guidelines?

\section{Conclusion and future perspectives}

Non-invasive, image-facilitated navigation for guided LV lead placement in CRT has been shown to be a clinically feasible approach to take full advantage of the method's potential. It is logistic and workup issues rather than a paucity of evidence, or technical constraints that actually impedes the concept from being implemented into routine practice. Although the data are not invariably convincing, there are specific populations that may particularly profit from tailored implantation: these are patients with ICM, non-specific conduction impairment, a history of previously failed implantation, or those who initially failed to respond. From the clinical standpoint, it must finally be acknowledged that alternative methods of stimulation, such as multipoint or multisite pacing, LV endocardial pacing, either conventional or leadless (wireless), may be able to compensate for suboptimal LV lead placement. At the same time, these techniques will allow for virtually unrestricted lead positioning - an advantage that can only be fully exploited through tailored implantation.

\section{Conflict of interests}

No relationships with industry and other entities are declared. The authors have no conflict of interest to be declared. No financial support was received.

\section{References}

Abraham WT, Fisher WG, Smith AL, Delurgio DB, Leon AR, Loh E, et al. (2002). Cardiac resynchronization in chronic heart failure. N Engl J Med 346(24): 1845-1853. DOI: 10.1056/ NEJMoa013168.

Adelstein EC, Saba S (2007). Scar burden by myocardial perfusion imaging predicts echocardiographic response to cardiac resynchronization therapy in ischemic cardiomyopathy. Am Heart J 153(1): 105-112. DOI: 10.1016/j.ahj.2006.10.015.

Adelstein EC, Tanaka H, Soman P, Miske G, Haberman SC, Saba SF, Gorcsan J 3rd (2011). Impact of scar burden by single-photon emission computed tomography myocardial perfusion imaging on patient outcomes following cardiac resynchronization therapy. Eur Heart J 32(1): 93-103. DOI: 10.1093/eurheartj/ehq389.

Auricchio A, Prinzen FW (2011). Non-responders to cardiac resynchronization therapy: The magnitude of the problem and the issues. Circ J 75(3): 521-527. DOI: 10.1253/circj.cj-10-1268. 
Becker M, Zwicker C, Kaminski M, Napp A, Altiok E, Ocklenburg C, et al. (2011). Dependency of cardiac resynchronization therapy on myocardial viability at the LV lead position. JACC Cardiovasc Imaging 4(4): 366-374. DOI: 10.1016/j.jcmg.2011.01.010.

Behar JM, Rajani R, Pourmoteza A, Preston R, Razeghi O, Niederer S, et al. (2017a). Comprehensive use of cardiac computed tomograpy to guide left ventricular lead placement in cardiac resynchronization therapy. Heart Rhythm 14(9): 1364-1372. DOI: 10.1016/j.hrthm.2017.04.041.

Behar JM, Mountney P, Toth D, Reiml S, Panayiotou M, Brost A, et al. (2017b). Real-time X-MRI-guided left ventricular lead implantation for targeted delivery of Cardiac Resynchronization Therapy. JACC Clin Electrophysiol 3(8): 803-814. DOI: 10.1016/j. jacep.2017.01.018.

Bertini M, Mele D, Malagù M, Fiorencis A, Toselli T, Casadei F, et al. (2016). Cardiac resynchronization therapy guided by multimodality cardiac imaging. Eur J Heart Fail 18(11): 13751382. DOI: $10.1002 /$ ejhf.605.

Bleeker GB, Kaandorp TA, Lamb HJ, Boersma E, Steendijk P, de Roos A, et al. (2006). Effect of posterolateral scar tissue on clinical and echocardiographic improvement after cardiac resynchronization therapy. Circulation 113(7): 969-976. DOI: 10.1161/CIRCULATIONAHA.105.543678.

Brignole M, Auricchio A, Baron-Esquivias G, Bordachar P, Boriani G, Breithardt OA, et al. (2013). 2013 ESC Guidelines on cardiac pacing and cardiac resynchronization therapy: the Task Force on cardiac pacing and resynchronization therapy of the European Society of Cardiology (ESC). Developed in collaboration with the European Heart Rhythm Association (EHRA). Eur Heart J 34(29): 2281-329. DOI: 10.1093/eurheartj/eht150.

Butter C, Auricchio A, Stellbrink C, Fleck E, Ding J, Yu Y, et al. (2001). Pacing Therapy for Chronic Heart Failure II Study Group. Effect of resynchronization therapy stimulation site on the systolic function of heart failure patients. Circulation 104(25): 3026-3029. DOI: 10.1161/hc5001.102229.

Chalil S, Stegemann B, Muhyaldeen SA, Khadjooi K, Foley PW, Smith RE, Leyva F (2007). Effect of posterolateral left ventricular scar on mortality and morbidity following cardiac resynchronization therapy. Pacing Clin Electrophysiol 30(10): 1201-1209. DOI: 10.1111/j.1540-8159.2007.00841.x.

Chen J, Henneman MM, Trimble MA, Bax JJ, Borges-Neto S, Iskandrian $\mathrm{AE}$, et al. (2008). Assessment of left ventricular mechanical dyssynchrony by phase analysis of ECG-gated SPECT myocardial perfusion imaging. J Nucl Cardiol 15(1): 127-136. DOI: 10.1016/j.nuclcard.2007.11.004.

Christiaens L, Ardilouze P, Ragot S, Mergy J, Allal J (2008). Prospective evaluation of the anatomy of the coronary venous system using multidetector row computed tomography. Int J Cardiol 126(2): 204-208. DOI: 10.1016/j.ijcard.2007.03.128.

Daubert C, Behar N, Martins RP, Mabo P, Leclercq C (2017). Avoiding non-responders to cardiac resynchronization therapy: a practical guide. Eur Heart J 38(19): 1463-1472. DOI: 10.1093/eurheartj/ ehw270.

Dekker AL, Phelps B, Dijkman B, van der Nagel T, van der Veen FH, Geskes GG, Maessen JG (2004). Epicardial left ventricular lead placement for cardiac resynchronization therapy: optimal pace site selection with pressure-volume loops. J Thorac Cardiovasc Surg 27(6): 1641-1647. DOI: 10.1016/j.jtcvs.2003.10.052.

Döring M, Braunschweig F, Eitel C, Gaspar T, Wetzel U, Nitsche B (2013). Individually tailored left ventricular lead placement: lessons from multimodality integration between threedimensional echocardiography and coronary sinus angiogram. Europace 15(5): 718-727. DOI: 10.1093/europace/eus396.

Groenning BA, Nilsson JC, Sondergaard L, Frits-Hansen T, Larsson HB, Hildebrand PR (2000). Antiremodeling effects on the left ventricle during beta-blockade with metoprolol in the treatment of chronic heart failure. J Am Coll Cardiol 36(7): 2072-2080. DOI: 10.1016/s0735-1097(00)01006-8.

Jongbloed MR, Lamb HJ, Bax JJ, Schuijf JD, de Roos A, van der Wall EE, Schalij MJ (2005). Noninvasive visualization of the cardiac venous system using multislice computed tomography. J Am Coll Cardiol 45(5): 749-753. DOI: 10.1016/j. jacc.2004.11.035.
Khan FZ, Virdee MS, Gopalan D, Rudd J, Watson T, Fynn SP, Dutka DP (2009). Characterization of the suitability of coronary venous anatomy for targeting left ventricular lead placement in patients undergoing cardiac resynchronization therapy. Europace 11(11): 1491-1495. DOI: 10.1093/europace/eup292.

Khan FZ, Virdee MS, Palmer CR, Pugh PJ, O’Halloran D, Elsik M, et al. (2012). Targeted left ventricular lead placement to guide cardiac resynchronization therapy: the TARGET study: a randomized, controlled trial. J Am Coll Cardiol 59(17): 15091518. DOI: 10.1016/j.jacc.2011.12.030.

Kutyifa V, Zareba W, McNitt S, Singh J, Hall WJ, Polonsky S, et al. (2013). Left ventricular lead location and the risk of ventricular arrhythmias in the MADIT-CRT trial. Eur Heart J 34(3): 184-190. DOI: 10.1093/eurheartj/ehs334.

Laksman Z, Yee R, Stirrat J, Gula LJ, Skanes AC, Leong-Sit P, et al. (2014). Model-based navigation of left and right ventricular leads to optimal targets for cardiac resynchronization therapy: a single centre feasibility study. Circ Arrhythm Electrophysiol 7(6): 1040-1047. DOI: 10.1161/CIRCEP.114.001729.

Lambiase PD, Rinaldi A, Hauck J, Mobb M, Elliott D, Mohammad S, et al. (2004). Non-contact left ventricular endocardial mapping in cardiac resynchronisation therapy. Heart 90(1): 44-51. DOI: 10.1136/heart.90.1.44.

Leyva F, Foley P, Chalil S, Ratib K, Smith RE, Prinzen F, Auricchio A (2011). Cardiac resynchronization therapy guided by late gadolinium-enhancement cardiovascular magnetic resonance. J Cardiovasc Magn Reson 13: 29. DOI: 10.1186/1532-429X13-29.

Ludwig DR, Menon PG, Schwartzman D (2015). Nuclear imageguided left ventricular pacing lead navigation feasibility of a new technique. J Interv Card Electrophysiol 44(3): 273-277. DOI: 10.1007/s10840-015-0046-9.

Ma YL, Shetty AK, Duckett S, Etyngier P, Gijsbers G, Bullens R, et al. (2012). An integrated platform for image-guided cardiac resynchronization therapy. Phys Med Biol 57(10): 2953-2968. DOI: $10.1088 / 0031-9155 / 57 / 10 / 2953$.

Mendoza DD, Joshi SB, Weissman G, Taylor AJ, Weigold WG (2010). Viability imaging by cardiac computed tomography. J Cardiovasc Comput Tomogr 4(2): 83-91. DOI: 10.1016/j.jcct.2010.01.019.

Pourmorteza A, Schuleri KH, Herzka DA, Lardo AC, McVeigh ER (2012). A new method for cardiac computed tomography regional function assessment: stretch quantifier for endocardial engraved zones (SQUEEZ). Circ Cardiovasc Imaging 5(2): 243-250. DOI: 10.1161/CIRCIMAGING.111.970061.

Saba S, Marek J, Schwartzman D, Jain S, Adelstein E, White P, et al. (2013). Echocardiography-guided left ventricular lead placement for cardiac resynchronization therapy: results of the Speckle Tracking Assisted Resynchronization Therapy for Electrode Region trial. Circ Heart Fail 6(3): 427-434. DOI: 10.1161/ CIRCHEARTFAILURE.112.000078.

Shetty AK, Duckett SG, Ginks MR, Ma Y, Sohal M, Bostock J, et al. (2013). Cardiac magnetic resonance-derived anatomy, scar, and dyssynchrony fused with fluoroscopy to guide LV lead placement in cardiac resynchronization therapy: a comparison with acute haemodynamic measures and echocardiographic reverse remodeling. Eur Heart J Cardiovasc Imaging 14(7): 692-699. DOI: 10.1093/ehjci/jes270.

Singh JP, Houser S, Heist EK, Ruskin JN (2005). The coronary venous anatomy: a segmental approach to aid cardiac resynchronization therapy. J Am Coll Cardiol 46(1): 68-74. DOI: 10.1016/j.jacc.2005.04.017.

Singh JP, Klein HU, Huang DT, Reek S, Kuniss M, Quesada A (2011). Left ventricular lead position and clinical outcome in the multicenter automatic defibrillator implantation trialcardiac resynchronization therapy (MADIT-CRT) trial. Circulation 123(11): 1159-1166. DOI: 10.1161/ CIRCULATIONAHA.110.000646.

Sommer A, Kronborg MB, Nørgaard BL, Gerdes C, Mortensen PT, Nielsen JC (2014). Left and right ventricular lead positions are imprecisely determined by fluoroscopy in cardiac resynchronization therapy: a comparison with cardiac computed tomography. Europace 16(9): 1334-1341. DOI: 10.1093/europace/ euu056. 
Sommer A, Kronborg MB, Nørgaard BL, Poulsen SH, Bouchelouche K, Böttcher M, et al. (2016). Multimodality imaging-guided left ventricular lead placement in cardiac resynchronization therapy: a randomized controlled trial. Eur J Heart Fail 18(11): 1365-1374. DOI: 10.1002/ejhf.530.

Spencer JH, Larson AA, Drake R, Iaizzo PA (2014). A detailed assessment of the human coronary venous system using contrast computed tomography of perfusion-fixed specimens. Heart Rhythm 11(2): 282-288. DOI: 10.1016/j.hrthm.2013.10.038.

Tournoux FB, Manzke R, Chan RC, Solis J, Chen-Tournoux AA, Gérard O, et al. (2007). Integrating functional and anatomical information to facilitate cardiac resynchronization therapy. Pacing Clin Electrophysiol 30(8): 1021-1022. DOI: 10.1111/j.15408159.2007.00803.x.

Van de Veire NR, Schuijf JD, De Sutter J, Devos D, Bleeker GB, de Roos A, et al. (2006). Non-invasive visualization of the cardiac venous system in coronary artery disease patients using 64-slice computed tomography. J Am Coll Cardiol 48(9): 1832-1838. DOI: 10.1016/j.jacc.2006.07.042.

Ypenburg C, Roes SD, Bleeker GB, Kaandorp TA, de Roos A, Schalij MJ, et al. (2007a). Effect of total scar burden on contrastenhanced magnetic resonance imaging on response to cardiac resynchronization therapy. Am J Cardiol 99(5): 657-660. DOI: 10.1016/j.amjcard.2006.09.115.

Ypenburg C, Schalij MJ, Bleeker GB, Steendijk P, Boersma E, Dibbets-Schneider P, et al. (2007b). Impact of viability and scar tissue on response to cardiac resynchronization therapy in ischaemic heart failure patients. Eur Heart J 28(1): 33-41. DOI: 10.1093/eurheartj/ehl379.

Zhou W, Hou X, Piccinelli M, Tang X, Tang L, Cao K, et al. (2014). $3 D$ fusion of $L V$ venous anatomy on fluoroscopy venograms with epicardial surface on SPECT myocardial perfusion images for guiding CRT LV lead placement. JACC Cardiovasc Imaging 7(12): 1239-1248. DOI: 10.1016/j.jcmg.2014.09.002. 